\section{Jahre Praxis}

sind auch 40 Jahre

Familien- und

\section{Medizingeschichte}

\author{
M. Schillig
}

Erinnerungen sind ein goldener Rahmen, der jedes Bild freundlicher macht.

Carl Zuckmayer

\section{Sind Heiligenscheine 100karätig?}

Von den Diakonissen zum Dienen erzogen, war es für uns Schwestern eine Tatsache, dass die Ärzte auf einem Podest standen und unfehlbar wie der Papst waren. Professor Fanconi unterstützte diese Meinung auch noch. Später versuchte Frau Dr. Hartmann meine Idealvorstellungen etwas zu relativieren. Wenn auch die Heiligenscheine etwas verblassten, hatte ich vor jeder Chefvisite ein ehrfürchtiges Gefühl. Waren die Kurven nachgeführt, lagen die Kinder fast in Achtungsstellung unter den glattgestrichenen Decken und war auch meine Schürze fleckenlos? Einmal konnte die Oberschwester im letzten Moment mit einer Tour Gipsbinden den Spruch "Schwester Maja und Dr. Schillig" auf dem Unterschenkelgips eines Knaben überdecken.

Ich genoss als Kinderschwester auf der Chirurgie eine gewisse Vorzugsstellung. Drei Zimmer auf der Frauenabteilung mit max. 12 Kindern waren mein Reich. Ich konnte die Zeit mehr oder weniger selber einteilen. Mussten am Morgen viele Kinder gewaschen werden, war ich eben etwas früher auf der Abteilung. Der Feierabend begann mit dem Antritt der Nachtschwester und wenn alle Kinder versorgt waren.

1955 war das "Kanti» wieder mein Zuhause. Zunehmende Erfahrungen liessen einem nicht so schnell aus der Ruhe bringen. Die Weiterbildung bestand darin, dass man sich fortlaufend mit den Neuerungen bekannt machte und das waren nicht wenige, denn die Medizin befand sich in einer grossen und schnellen Entwicklung.

Noch war das Kanti überschaubar. Die gemeinsamen Essenszeiten und die internen Wohngelegenheiten ergaben gute Gespräche, manchmal auch Klatsch, aber man fühlte sich nie allein gelassen und schloss viele Freundschaften.

Korrespondenz:

Maja Schillig

Tersierstrasse 202

CH-7220 Schiers

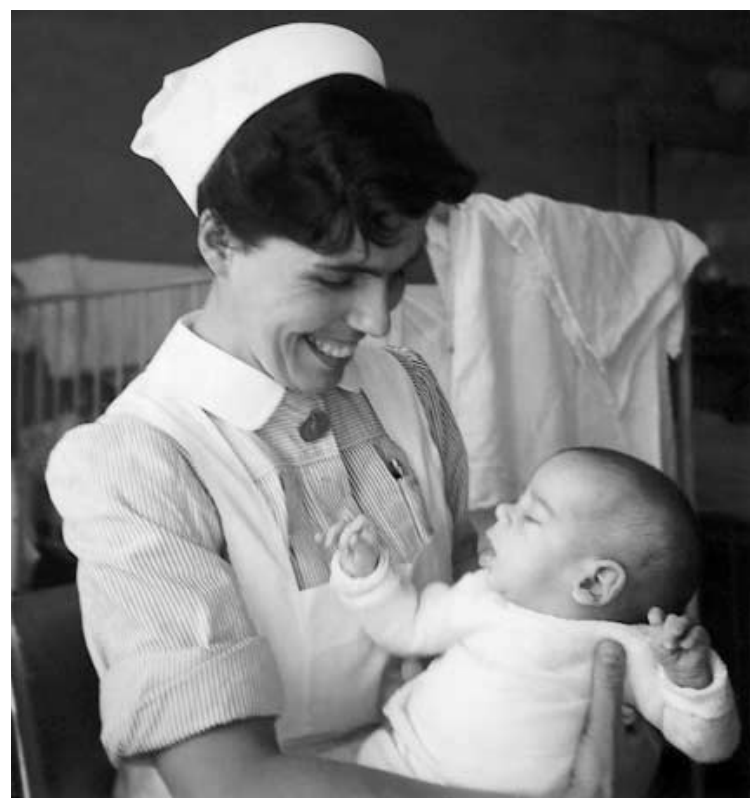

Maja im Element.

Die Freizeit nutzten wir hin und wieder gemeinsam, aber die eigenen vier Wände waren auch sehr wertvoll.

Es herrschten strenge Vorschriften: die Schwestern hatten auf dem Stockwerk der Assistenten nichts zu suchen, ebenso wenig durfte sich ein Mann im Schwesternbereich sehen lassen. Sehr zu unserer Missbilligung waren die Laborantinnen von diesen strengen Regeln ausgeschlossen.

Mit Martin Allgöwer und nachdem die Diakonissen diese Aussenstation gekündigt hatten, begann eine neue Ära. Dank seiner umgänglichen Art konnte der Chef vieles bewirken. Mit dem Verwalter und Dr. Markoff, dem medizinischen Chef, verstand er sich besser, als sein Vorgänger, und er konnte viele Neuerungen einführen. Es herrschte eine offene Atmosphäre und Martin Allgöwer fand auch hin und wieder Zeit zum Skifahren mit einem Assistenten. Auf dem Parkplatz hinter dem Spital standen nur zwei Autos, das des Chefs und des Oberarztes. Mit einem maximalen Lohn von Fr.1000.- konnte sich kein Assistent ein solches leisten. Die Ärzte verdienen heute besser, aber der Arbeitstag ist hektischer und die Patienten kritischer geworden. War das Gold vom Heiligenschein nur Blattgold oder ist es sonst abgebröckelt?

\section{Die Suche}

Unsere Wohnung befand sich an der Quaderstrasse in Chur über dem Migros und vis-à-vis von einem Kino, welches an warmen Sommerabenden die Türe des Vorführungsraums offen liess, so dass wir zu mehr oder weniger schönen Musikdarbietungen kamen.

Als das Jahr zu Ende war, zügelten wir nach Luzern. "Geniesst die Assistentenzeit ohne eigene Praxis", dies war der oft gehörte Ratschlag und das 


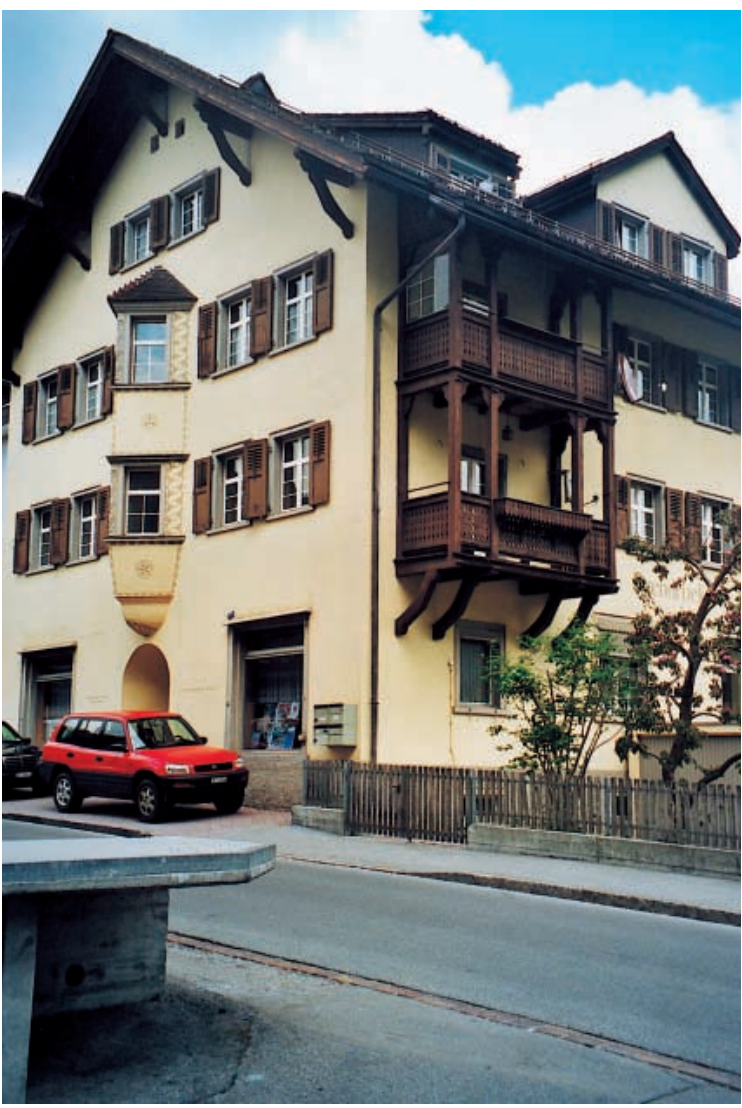

Wohnung (bis 1963).

taten wir auch. Luzern gab einem das Gefühl, in den Ferien zu sein. $\mathrm{Zu}$ dieser Zeit hätte ich keine Möglichkeit gehabt, als Kinderschwester eine Teilzeitarbeit zu finden, erst mit dem Schwesternmangel in späteren Jahren erkannte man diese Lösungen.

Alex hatte jeden zweiten Tag und jedes zweite Wochenende Dienst, aber in der übrigen Zeit konnten wir doch einiges unternehmen, so fuhren wir aus dem Nebel hinaus ins Eigental, machten im Sommer

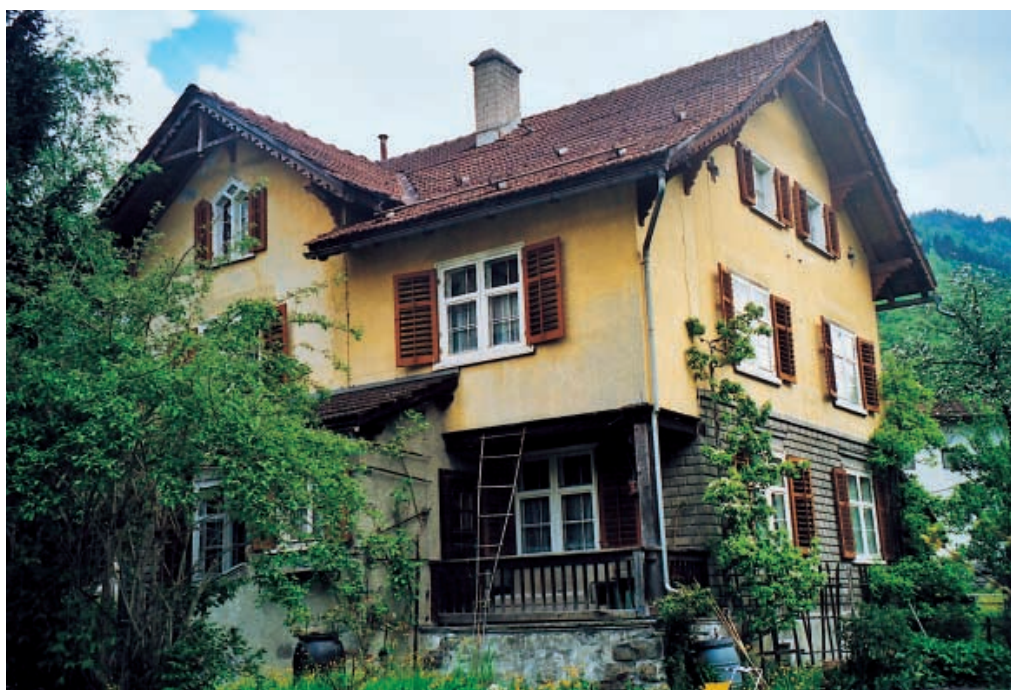

Praxis bis 1967. einen Ausflug auf den Pilatus oder genossen den See. Sogar für Englischstunden reichte unsere Energie. So unabhängig sollten wir in den nächsten Jahren nicht mehr sein. Bald mussten wir im "gelben Heft" nach Praxisangeboten suchen. Im Entlebuch hätte es uns gefallen, aber die Kosten für das Haus und die Praxis waren zu hoch. Auch bin ich nachträglich sehr zufrieden, dass wir uns nicht in Saanen niederliessen. Die Nobelkundschaft wäre nicht nach unserem Geschmack gewesen und die Familie doch reichlich weit weg.

Alex wünschte sich eine Praxis in den Bergen und eine ländliche Bevölkerung. Wir reisten herum und hatten genug Zeit. Auch Heiden und Andeer kamen noch in Frage. In Korea wurde immer Schweizer Militär für Bewachungsaufgaben gesucht.

\section{Sind Zufälle Zufälle?}

In dieser Zeit benötigte Mama ärztliche Hilfe von Dr. Caprez in Chur. Seine "Tonsillenkinder" habe ich lange Zeit gepflegt. Im Gespräch mit ihm erwähnte Mama unsere Praxissuche. Dr. Caprez hatte gehört, dass in Schiers ein Arzt benötigt werde. Die Krankenkasse Helvetia hatte den Auftrag, für den verstorbenen Dr. Zimmerli einen Nachfolger zu finden. Nun sollten wir uns dort melden. Wieder Graubünden, altbekannte, gewohnte Umgebung, das Spital Chur in der Nähe und auch die Familie gut erreichbar, das klang verlockend.

An einem schönen Junitag fuhren wir los. Bei schönem Wetter ist es überall schön!! Wir trafen uns mit dem Krankenkassenpräsidenten und Dr. Heinz, welcher noch einige alte Patienten betreute (heute nach 40 Jahren sind wir in der gleichen Lage) und von welchem die Leute sagten: "der laidi Heinz ist immer in der Ferien». Der Krankenkassenverantwortliche zeigte sich skeptisch betreffs Alex Konfession, aber ein Katholik sei immer noch besser als einer aus den Balkanstaaten.

Die Praxisübernahme hing auch noch von den Wohnmöglichkeiten ab. Wir hatten die Wahl zwischen einer in der Buchdruckerei oder im Haus neben der heutigen FEG. So entschieden wir uns für die Bahnhofstrasse. Diese Wohnung war sehr grosszügig, mit einer neuerstellten Zentralheizung, einer grossen Stube mit Kachelofen und sie kostete Fr. 180.-- Im 1. Stock wohnte Oberst Thöny und auf seinen Wunsch (oder Befehl) sollte die Haustüre nachts nicht abgeschlossen werden, weil der Einbrecher sie sonst nur beschädigen würde.

Dr. Zimmerli war vorgängig Chefarzt im Spital Schiers gewesen, wurde aber aus politischen Gründen weggewählt. Bevor er seine Praxis an der hinteren Bahnhofstrasse eröffnete, praktizierte er im zweiten Stock in der Buchdruckerei. Als wir später dort einzogen, zeugten immer noch abgeschlagene Stellen im Brünneli von seiner Tätigkeit, weil er nach dem Zahnziehen die Zange dort hinein warf. Dem Spital zum Trotz soll er auch in der Praxis operiert haben. Viele Patienten kamen erst nach dem Eindunkeln, 
damit niemand kontrollieren konnte, $\mathrm{zu}$ welchem Arzt sie gingen.

Solche Probleme konnte es für uns nicht mehr geben, denn wir waren völlig unbelastet und die Konfession schien den Patienten keine Probleme zu machen.

\section{Tarife, Krankenscheine und alles "Drumherum»}

In den ersten Tagen der Praxiseröffnung (der erste mutige Patient kam von Schuders), besuchte uns Hans Schnyder, der einzige Kollege aus dem Prättigau. Er überliess Alex einige Patienten aus Schiers und Umgebung. Einige von auswärtigen Naturheilern betreute Kranke fanden zurück zur Schulmedizin. Hans und Alex einigten sich auf einen Notfalldienst, wobei Hans sowieso im Winter jeden Sonntag zu Hause blieb, weil die verletzten Skifahrer von der Parsenn weiterhin in Küblis landeten. Das Spital brachte etwas Erleichterung in diesen Dienstplan.

Mir erklärte Hans das Abrechnungswesen, wobei er mich mit den auswendig aufgezählten Tarifpositionen verblüffte. Auch ich sollte dies mit den Jahren noch lernen. Einige Erfahrungen brachte Alex bereits aus seinen Praxisvertretungen mit. Im ersten Halbjahr machte ich die Laborarbeiten mit Kenntnissen, die ich bei Hedi Flach erworben hatte. Den ersten Telefonapparat musste Alex auf der Telefondirektion in Chur abholen, aber nicht immer freute uns dessen Läuten. Nach Ansicht von Alex ist es keine gute Lösung, wenn die Arztfrau in der Praxis mithilft, weil sich die Berufsprobleme noch mehr ins Privatleben mischen. So wechselte ich zur «Nur-Hausfrau».

Die Praxisräume waren im Haus Dr. Zimmerli. Die Küche war Labor, die Stube das Sprechzimmer. Durch den Anbau kam man ins Wartezimmer (ein besseres "Schöpfli»). Unsere Neuanschaffungen bestanden aus Röntgenapparat, Untersuchungstisch, Medikamentenschrank und Schreibtisch. Im Winter musste vom Labor aus die Zentralheizung mit Kohle gefeuert werden und Frau Zimmerli fand es nicht nötig, dass schon am Vormittag geheizt wurde.

Einen abgewetzten, wahrscheinlich bequemen, allseits bekannten Patientenstuhl und medizinische Sachbücher wollte Alex zu Frau Zimmerlis Leidwesen nicht übernehmen, hingegen konnte er die Zahnzangen in den ersten Jahren noch gebrauchen.

Bis 1967 waren wir mit diesen Räumlichkeiten zufrieden, aber als dann die "Valina" neben der Post gebaut wurde, ergab sich die Möglichkeit, eine modernere Praxis einzurichten. Nur das alte Mobiliar wurde gezügelt.

\section{"Perlen" und cand. med.}

Sie waren fast immer "Perlen», unsere Arztgehilfinnen. Wilma, die erste, kam von Appenzell und es war ihre erste Stelle nach der Ausbildung in der Arztgehilfinnenschule St. Gallen. Das erste Halbjahr galt als Praktikum. Sie wohnte bei Frau Dr. Zimmerli und wurde bei uns verpflegt. Anfangs blieb sie auch an den Wochenenden in Schiers und ihre Mutter legte mir ans Herz, dass ich sie am Sonntag in die Kirche schicken sollte. Die Geburt von Katrin freute sie so, dass Frau Zimmerli sie fragte, wer eigentlich geboren habe. Die meisten dieser jungen Mädchen blieben ein bis zwei Jahre bei uns. Mit den Haushalthilfen verband sie oft eine schöne Freundschaft, so machte Renate mit Annadorli aus Fanas und Alex eine Tour auf die Chesa-Plana.

Es gab auch ELA-Schüler, welche gerne am Nachmittag das Labor besuchten und dort manchmal auch behilflich waren, besonders als Renate mit einem Gips herumhumpelte. "Fehlbesetzungen" gab es selten, nur einmal verschwanden nicht-differenzierte Blutbilder in einer Schublade und falsche Medikamente wurden verschickt. Das war wahrscheinlich das einzige Mal, dass Alex die Geduld verlor, der Schublade einen Tritt versetzte und das Mädchen auf der Stelle entliess. Eine Arztgehilfin vermisste das tägliche Lob vom Chef, aber sonst waren sie doch alle sehr einsatzfreudig, auch bei einer langen Arbeitszeit, denn an zwei Abenden mussten sie bis etwa 21 Uhr anwesend sein. Heute würde sich sicher eine Kommission für Menschenrechte einschalten! Mit ihnen zusammen betreute ich auch die cand. med., welche während Alex' Militärdiensten oder seinen Reisen die Praxis führen musste. Einem musste Renate Kakao kochen, weil die Verantwortung sich auf sein Innenleben auswirkte. Mit der Kost konnte ich auch nicht alle erfreuen oder der Kinderlärm störte sie.

Modest Cahannes brauchte Dudu zur "Belastung" hinten im VW bei einer winterlichen Fahrt nach Pusserein. Hier kann ich auch erwähnen, dass im ersten Winter die Strasse nach Schuders nicht gepflügt war, erst mit Unterstützung des Veterinärs konnte die Gemeinde von der Schneeräumung überzeugt werden. Da war unter den verschiedenen Assistenten auch Herr Kolb aus Österreich, welcher von Alex jun. beneidet wurde, weil er vier Zucker in den Tee geben durfte. Das Trompetenspiel eines anderen animierte ihn zur späteren Erlernung dieses Instrumentes. Ehrlich gesagt, ich begrüsste die Praxiseröffnung von Walter Studer in Grüsch, endlich hatte dieser Hotelbetrieb ein Ende, die Verköstigung, besonders am Abend, war vereinfacht und wir waren wieder unter uns.

\section{"Da wohn ich lieber unter den Lawinen»??}

Eine Landpraxis erfordert auch einige Fitness. Von den Lawinen wurden wir zwar nie bedroht, aber die Wege und Strassen boten hin und wieder einige Schwierigkeiten. Wie ich schon erwähnte, war im ersten Winter die Strasse nach Schuders nicht offen, denn die Bauern brauchten für ihre Transporte einen Schlitten. Ich kann mich nicht erinnern, dass Alex mit den Skiern unterwegs war, nur einmal für eine Zeitungsreportage in Stels. In Rekordzeit konnte er die Schneeketten montieren. Neben der medizinischen Ausrüstung gehörte auch eine Lawinenschaufel ins 
Auto, denn es kam hin und wieder vor, dass Schneerutsche einem an der Weiterfahrt hinderten. Die Feldstrasse war ein einfaches Schottersträsschen ohne Beleuchtung und bei Dunkelheit für den Fussgänger nicht gut zu finden. Einmal weckte mich ein spulendes Geräusch, und im Pyjama unter dem Mantel half ich das Auto aus dem Graben zu schieben.

Gigi und Piet mussten im März das Auto beim Spital parkieren, weil auf der tief verschneiten Strasse an kein Weiterkommen zu denken war. Die Feldstrasse führte nur bis ins Tersier, es waren meist keine Autos unterwegs, dafür aber Hirsche und Rehe. Die Berglandschaft brachte auch schöne Momente, z.B. eine Fahrt bei Sonnenaufgang nach Munt oberhalb Fanas, oder ein Heliflug nach Furna. Ein Stück Käse war das Geschenk einer Familie in Salfsch, wohin Alex gerufen wurde. Die Tochter des Patienten kam ihm mit einer Laterne im Tobel entgegen, damit er den Weg finden konnte.

Im Frühling schmilzt das Eis auf den Strassen, aber in der Nacht bilden sich wieder Eisblasen. Das hätte 1979 für Alex schlimm enden können. Auf einer Nachtfahrt nach Schuders rutschte das Auto, überschlug sich und landete auf dem unteren Strassenabschnitt. Gott sei Dank wurde der Unfall vom vorausfahrenden Fahrer bemerkt, welcher Alex ins Tal hinunter brachte. Im Spital konnten nach einer Untersuchung keine äusseren Verletzungen festgestellt werden. Der Kieferbruch, vom Zahnarzt diagnostiziert, musste später in Zürich operiert werden. Nicht von einem Patienten, aber durch ein afghanisches Getränk wurde Alex angesteckt und «sollte» wegen einer
Gelbsucht das Bett hüten. Er verbrachte die meiste Zeit im Lehnstuhl vor dem Fernseher und verfolgte die olympischen Spiele. Als ich an einem Pullover strickte, meinte er, der würde sich für ihn nicht mehr lohnen.

Die Nachtbesuche und auch die regulären Visiten nahmen ab, als die Bevölkerung sich immer mehr motorisierte. Der erste Autobesitzer in Fanas wurde von den Einheimischen restlos bewundert und es war Annadorli, welche mir dieses wichtige Ereignis schilderte. Mit der Zeit wurde auch der Notfalldienst besser organisiert. Anfangs war es keine Seltenheit, dass an einem Nachmittag bis zu 12 Patienten besucht werden mussten. Auf die Handyerfindung mussten wir noch einige Jahre warten. So war z.B. der Konsumverwalter in Fanas eine Ansprechstelle: die Arztgehilfin hatte die Besuchsliste und konnte jeweils dorthin oder zu einem Patienten telefonieren. Sonntagsdienst bedeutete auch für mich Pikett. Oft habe ich auf Autogeräusche oder auf die Lichter gewartet.

Dasselbe viele Jahre später nochmals, wenn die Söhne unterwegs waren.

Nun sind viele Kollegen im Tal verteilt und der Notfalldienst entfällt auf die "Jungen». Die Menschen erzählen immer noch von Dr. Flury, welcher mit dem Ross unterwegs war, der alte Dr. Schnyder las die Zeitung auf dem Marsch nach St. Antönien. Dr. Mark holte zusammen mit der Hebamme eine Wöchnerin in Schuders ab, letztere zog es vor, bei einer gefährlichen Strassenstelle von der Bahre abzusteigen. Wenn ich diese Zeilen schreibe, scheinen mir die von uns erlebten Ereignisse fast so weit weg. 